\title{
EVALUACIÓN DE LA SOSTENIBILIDAD EN EL SECTOR SOCIOTURÍSTICO DE FUERTEVENTURA (ISLAS CANARIAS)
}

\author{
Isabel Banos-González* \\ Universidad de Murcia \\ Julia Martínez-Fernández** \\ Fundación Nueva Cultura del Agua \\ Miguel Ángel Esteve*** \\ Universidad de Murcia \\ Pedro Pérez Cutillas**** \\ Universidad de Murcia
}

\section{RESUMEN}

Como herramienta para la evaluación cuantitativa del impacto turístico en sistemas socioecológicos insulares, en este trabajo se presenta el modelo dinámico de sostenibilidad de Fuerteventura. Una vez calibrado para el período 1996-2011, ha permitido una mejor comprensión de las interacciones dinámicas entre los indicadores de sostenibilidad y otros factores en la isla. También ha permitido analizar el comportamiento de algunos de esos indicadores bajo distintos escenarios y medidas de gestión para el periodo 2012-2025. Los resultados reflejan una influencia elevada de determinadas medidas de control relativas a la creación de nuevos alojamientos turísticos sobre la evolución de la sostenibilidad en la isla.

Palabras clave: escenarios, indicadores, modelos dinámicos, turismo, sostenibilidad.

Fecha de recepción: 11 de enero de 2018

Fecha de aceptación: 22 de mayo de 2018

* Departamento de Didáctica de las Ciencias Experimentales. Facultad de Educación. Universidad de Murcia.30100 Espinardo, MURCIA (España).E-mail: ibbg1@um.es

** Dirección Técnica Fundación Nueva Cultura del Agua. C/ Pedro Cerbuna, 12, 4 dcha, 50009 ZARAGOZA (España).E-mail: julia@fnca.eu

*** Departamento de Ecología e Hidrología. Facultad de Biología. Universidad de Murcia. 30100 Espinardo, MURCIA (España).E-mail: maesteve@um.es

**** Departamento de Geografía. Facultad de Letras. Universidad de Murcia. 30001 MURCIA (España). E-mail: pedrope@um.es 


\title{
Assessment of sustainability in the socio-tourist sector of Fuerteventura (The Canary Islands)
}

\begin{abstract}
In this work, Fuerteventura's dynamic sustainability model is applied as a tool for the quantitative evaluation of the tourism impact on insular socio-ecological systems. The model, calibrated for the period 1996-2011, has allowed a better understanding of the dynamic interactions between sustainability indicators and other factors on the island. Moreover, the analysis of the behavior of some of these indicators under different scenarios and management measures for the period 2012-2025. The results show a high influence of certain measures regarding the control on the creation of new tourist accommodation in the evolution of sustainability on the island.
\end{abstract}

Keywords: scenarios, indicators, dynamic models, tourism, sustainability.

\section{INTRODUCCIÓN}

Los sistemas socioecológicos (SSE) se pueden definir como sistemas integrados de ecosistemas y sociedades humanas en los que se produce una interacción constante entre ellos (Anderies et al., 2004; Halliday y Glaser, 2011). En sistemas complejos como los SSE, las variables que lo forman no interactúan de forma lineal, sino que se caracterizan por sus mecanismos de refuerzo, ensamblando la fracción socioeconómica y la ecológica en patrones de co-evolución (Gunderson y Holling, 2002; Ostrom, 2009). En este contexto, un cambio en la dinámica socioturística, por ejemplo, se reconoce como un cambio sistémico, que surge de un evento externo o de un cambio gradual endógeno, provocando una transformación en las interacciones que caracterizan al sistema, y puede provocar cambios en el estado global (Filatova y Polhill, 2012). De acuerdo con Ropero et al. (2014), cuando se produce uno de estos eventos, el cambio puede propagarse a través de todo el sistema por medio de interacciones de causa-efecto entre los componentes del SSE, que se relacionan formando una entidad compleja y dinámica, por lo que su análisis requiere de un enfoque holístico (Hodbod y Adger, 2014).

La sostenibilidad es una concepción transitoria, un objetivo eventual que puede ser mejorado continuamente a medida que aumenta nuestra comprensión del sistema (Ko, 2005; Hjorth y Bagheri, 2006). El concepto de sostenibilidad obtuvo su reconocimiento oficial en 1987 con el informe Brundtland, definiéndolo como "el desarrollo que satisface las necesidades de la generación actual sin comprometer la capacidad de la generación futura para que pueda asegurar sus propias necesidades" (WCED, 1987). Las tendencias insostenibles en la evolución de los sistemas sociales y ecológicos han estimulado la búsqueda de nuevos enfoques para comprender los complejos problemas del medio ambiente y el desarrollo (PNUMA, 2002). Existe una conciencia cada vez mayor sobre la necesidad de avanzar más rápidamente en la implementación de políticas y medidas más sostenibles. A pesar de esto, la aplicación real de políticas para la sostenibilidad en los SSE está bas- 
tante lejos de ser conseguida. Varias barreras y dificultades explican esta brecha entre el conocimiento y la acción, donde uno de los obstáculos principales es que, a pesar del creciente reconocimiento de las estrechas interdependencias entre los aspectos económicos, ambientales y sociales, en la práctica, estas dimensiones se consideran con frecuencia de forma separada. Una razón importante para que esto suceda reside en la complejidad de la propia naturaleza de los SSE; el resultado es que la información utilizada para gestionar los SSE se utiliza de forma inapropiada.

Así mismo, existe una falta de herramientas adecuadas para comprender, evaluar y comunicar las mejores opciones que promuevan sistemas más sostenibles. Por ello se debería favorecer una visión más integrada entre los gestores y responsables políticos, las partes interesadas y otros agentes, con respecto a cuestiones clave de sostenibilidad, basadas en sólidos conocimientos científicos. Finalmente, sería interesante la aplicación de todos estos enfoques en SSE reales, que puedan servir como sistemas piloto de demostración de estas políticas hacia la sostenibilidad; un papel que se reconoce explícitamente a las Reservas de la Biosfera.

Recientemente se ha detectado un mayor interés en la evaluación de la sostenibilidad en los sistemas socioecológicos insulares (Jørgensen, 2013; Petrosillo et al., 2013; Tsoukala et al., 2018). Esto puede deberse al amplio potencial de las islas como observatorios de sostenibilidad, donde se reconoce fácilmente la interacción entre los aspectos ecológicos y los procesos socioeconómicos, sobre todo, en aquellas donde la actividad turística juega un papel fundamental.

Sin embargo, la aplicación de este enfoque integral parece ser aún escasa en la práctica, particularmente en relación con la aplicación de enfoques cuantitativos (Anderies et al., 2004; Janssen, 2006). La mayoría de los estudios de sistemas socioecológicos se basan en perspectivas y metodologías cualitativas, pero, como Duffi et al. (2001) ha señalado, existe una "necesidad crítica" para el desarrollo de modelos cuantitativos que integren los sistemas físicos, biológicos y humanos.

Por todo ello, parece necesario el desarrollo y la aplicación de herramientas cuantitativas que permitan realizar un análisis integral de la sostenibilidad de sistemas socioecológicos reales, que puedan servir como laboratorios de demostración de cómo determinados escenarios y medidas de gestión pueden afectar a estos sistemas y analizar cuantitativamente su evolución en términos de sostenibilidad. En este sentido, la perspectiva holística de la dinámica de sistemas proporciona un marco adecuado para gestionar el cambio y la complejidad al comprender las reacciones dinámicas integradas en los sistemas complejos (Fong et al., 2009). Para Hjorth y Bagheri (2006), encontrar una manera de formular la realidad como un sistema y no como un conjunto de problemas independientes es esencial para hacer una buena planificación y gestión del territorio y los recursos. Por lo tanto, cualquier herramienta para simplificar el estudio del sistema complejo y predecir los efectos de los cambios en cualquiera de sus componentes es útil tanto en la investigación como en la gestión de los sistemas socioecológicos (Blanco, 2013).

En este trabajo se pretende demostrar la utilidad del uso de los modelos dinámicos en combinación con otras herramientas (SIG, paquetes estadísticos, indicadores de sostenibilidad) para la gestión sostenible del turismo en sistemas socioecológicos insulares. Así, los objetivos específicos de este trabajo son: i) desarrollar y verificar un modelo integral que 
incorpore los factores y procesos clave de un sistema socioecológico real, en este caso, el modelo de sostenibilidad de la isla de Fuerteventura (MSF) y que incluya los indicadores de sostenibilidad más relevantes en el modelo, como una herramienta para analizar los principales cambios y las interacciones entre estos factores e indicadores; ii) utilizar el modelo para analizar la vulnerabilidad de esta isla a cambios externos, como escenarios económicos y de cambio climático; iii) aplicar el modelo para evaluar cómo se comportan algunos de estos indicadores respecto a sus umbrales de sostenibilidad bajo un conjunto de medidas de gestión.

\section{2. ÁREA DE ESTUDIO}

El área de análisis se centra en la isla de Fuerteventura, con una superficie de 1.655 km2, situándose en la parte más oriental del archipiélago canario (Figura 1). Tiene un clima desértico (Torres Cabrera, 1995), con un promedio de precipitaciones anuales por debajo de los $120 \mathrm{~mm}$, que sustenta una débil cobertura de vegetación dominada por matorrales xerofíticos y pastizales anuales (Schuster et al., 2012). Una característica distintiva de su paisaje es la existencia de sistemas de dunas formados por 'jables' (tierra con orígenes del viento), que constituyen el hábitat de numerosos animales y plantas singulares. Las llanuras interiores constituyen otro elemento geomorfológico característico de la isla, donde se pueden encontrar muchas estructuras vinculadas al uso de los escasos recursos hídricos.

\section{Figura 1}

\section{LOCALIZACIÓN DE LA ISLA DE FUERTEVENTURA}
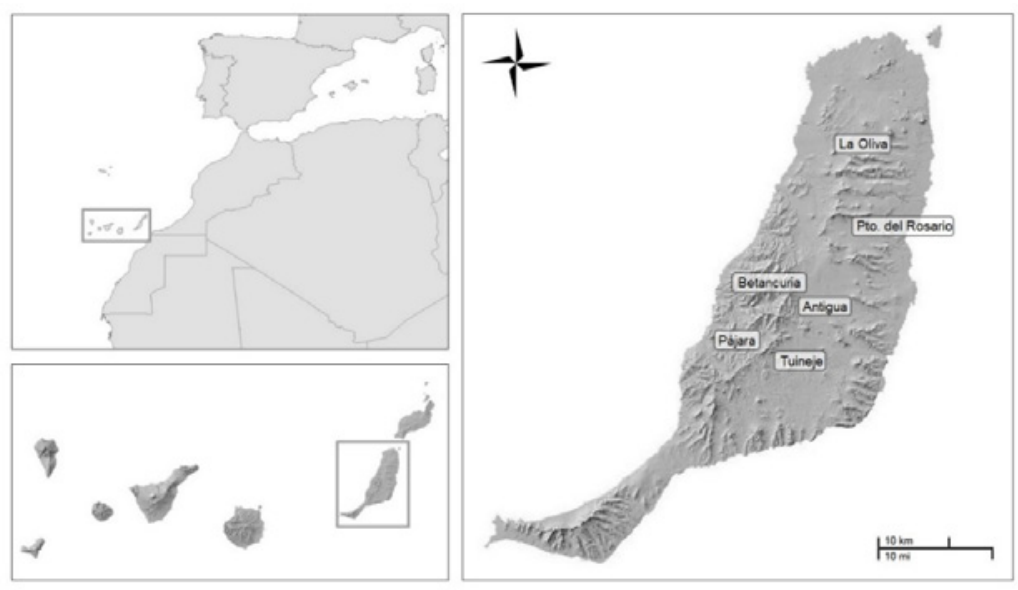

En las últimas décadas, el desarrollo turístico y el crecimiento de la población han provocado cambios en el uso del territorio, especialmente intensivos en algunas áreas costeras. Las actividades productivas tradicionales (ganadería y pesca artesanal), a pesar de que se han visto favorecidas por el auge del mercado turístico, han sido sustituidas 
principalmente por el turismo y actividades relacionadas. En casos como la agricultura de secano en gavias se ha producido el abandono de estas tierras, provocando la degradación de los paisajes rurales y la aceleración de los procesos de erosión del suelo. De manera paralela, Fuerteventura ha experimentado un espectacular crecimiento demográfico, con una tasa del 282\% desde 1990 hasta 2010, alcanzando más de cien mil habitantes en 2010 (ISTAC, 2012). Sin embargo, sigue siendo la isla con la densidad de población más baja de todo el archipiélago (40 habitantes / $\mathrm{km}^{2}$ en promedio entre 1991 y 2010, ISTAC, 2012).

Declarada Reserva de la Biosfera en 2009 (UNESCO, 2009), Fuerteventura es conocida por sus entornos áridos y por sus playas, que se pueden encontrar a lo largo de toda su costa, especialmente atractivas para el surf y otras actividades acuáticas (Santana-Jiménez y Hernández, 2011). Desde principios de los años 90, los alojamientos turísticos en la isla han crecido rápidamente tanto en hoteles como en alojamientos no hoteleros (Figura 2), con un escaso peso del turismo rural. Los turistas se concentran a lo largo de la costa, principalmente en los municipios de Pájara y La Oliva, propiciando un crecimiento rápido y desorganizado que ha ido convertido la isla en un destino para un turismo cada vez más masivo (Hernández-Luis et al., 2017). Este proceso está afectando de manera negativa a la imagen de la isla salvaje y pacífica, que fue su característica distintiva en comparación con otros destinos costeros con condiciones climáticas similares. A pesar de esto, el desarrollo turístico ha producido una mejora económica del archipiélago en la última década, deteniéndose en 2008 debido a la recesión económica internacional. El número de llegadas de turistas creció un 374\% de 1990 a 2010, cuando la isla recibió casi 1,5 millones de turistas extranjeros (Gobierno de Canarias, 2010).

Esta dinámica ha convertido al turismo en el principal motor de los cambios socioeconómicos y ambientales, provocando una notable transformación de la isla en los últimos años, lo que ha propiciado la aparición de nuevos desafíos socioecológicos, que deben abordarse con urgencia.

\section{Figura 2 \\ EVOLUCIÓN DE LOS ALOJAMIENTOS EN HOTELES Y NO HOTELEROS EN EL PERIODO 2000 A 2012}

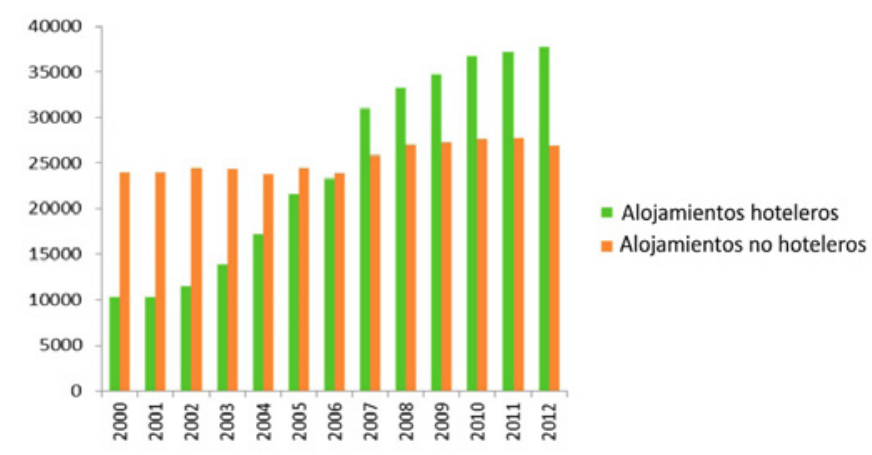

Fuente: Datos Gobierno de Canarias 2010. 


\section{METODOLOGÍA}

\subsection{Proceso de modelización}

La metodología de los sistemas dinámicos, desarrollada inicialmente por Forrester (1961), tiene un enorme potencial para su aplicación en la gestión sostenible de sistemas socioecológicos y la toma de decisiones, dada su capacidad para la comprensión del comportamiento dinámico del sistema y para entender las interrelaciones entre los distintos procesos. La simulación en este tipo de modelos dinámicos se lleva a cabo mediante ecuaciones diferenciales, utilizando fases de tiempo discretas. Además, los modelos de simulación dinámica permiten la evaluación del sistema bajo distintos escenarios futuros $\mathrm{y}$, por tanto, anticipar las consecuencias a medio y largo plazo de posibles medidas y estrategias de gestión (Kelly et al., 2013; Voinov y Shugart, 2013).

Por otro lado, los indicadores también se consideran un componente esencial de la evaluación de la sostenibilidad, puesto que representan una herramienta que permite reducir la complejidad de los procesos que representan, facilitando tanto su seguimiento como la comunicación con otros actores sociales afectados por dichos procesos y las decisiones que se toman al respecto (Singh, 2012; Poveda y Lipsett, 2014). Sin embargo, numerosos autores señalan la falta de utilidad real de los catálogos de indicadores en los procesos de adopción y evaluación de medidas de gestión aplicables a sistemas socioecológicos (Reed et al., 2006; Kajikawa et al., 2011). Por un lado, Von Geibler et al. (2011) apuntan la necesidad de que los indicadores sean dinámicos, es decir, que reflejen los continuos cambios e interacciones que experimentan los procesos que representan. Por otro lado, es necesario disponer de umbrales que permitan cuantificar si los cambios sufridos por los indicadores son aceptables o no en términos de sostenibilidad (Lancker and Nijkamp, 2000; Moldan et al., 2012).

En este trabajo se presenta un modelo de simulación dinámica de la Reserva de la Biosfera de Fuerteventura (el MSF), desarrollado para realizar un análisis integral de la sostenibilidad de la misma. El modelo MSF se aplica para explorar cómo una serie de indicadores de sostenibilidad integrados en el modelo interactúan con otras variables del sistema, mediante relaciones causales y circuitos de realimentación, así como la evolución de dichos indicadores bajo diferentes escenarios y medidas de gestión (Figura 3).

El primer paso para el desarrollo del MSF, enmarcado en la fase de conceptualización, fue la identificación de los temas más relevantes para la sostenibilidad en la isla, a partir de los resultados de la XI Conferencia Atlántica del Medio Ambiente (mayo de 2011). Con la implicación de los agentes sociales relevantes dentro de este SSE, se establecieron una serie de asuntos prioritarios y objetivos de sostenibilidad, también recogidos en el Plan de Acción de la Reserva de la Biosfera (AP, 2013). A partir de ahí, se definió el modelo conceptual y se identificaron un conjunto de indicadores de sostenibilidad, derivados también de una propuesta del Cabildo de Fuerteventura (Cáceres, 2010) que, una vez integrados, constituyen variables del propio modelo. A continuación, se determinaron los parámetros y se fueron formulando matemáticamente las distintas ecuaciones que conforman el modelo cuantitativo. El valor de los parámetros del modelo se determinó mayoritariamente a partir de la literatura científica, servidores estadísticos y otras fuentes 
documentales de los organismos de Fuerteventura. En los casos en los que no se ha contado con información, se ha efectuado un proceso de calibración automática por comparación con las series históricas disponibles (Oliva, 2003). Las ecuaciones se desarrollaron a partir de información de expertos y literatura científica específica, utilizando el software Vensim (Ventana Systems, 2011).

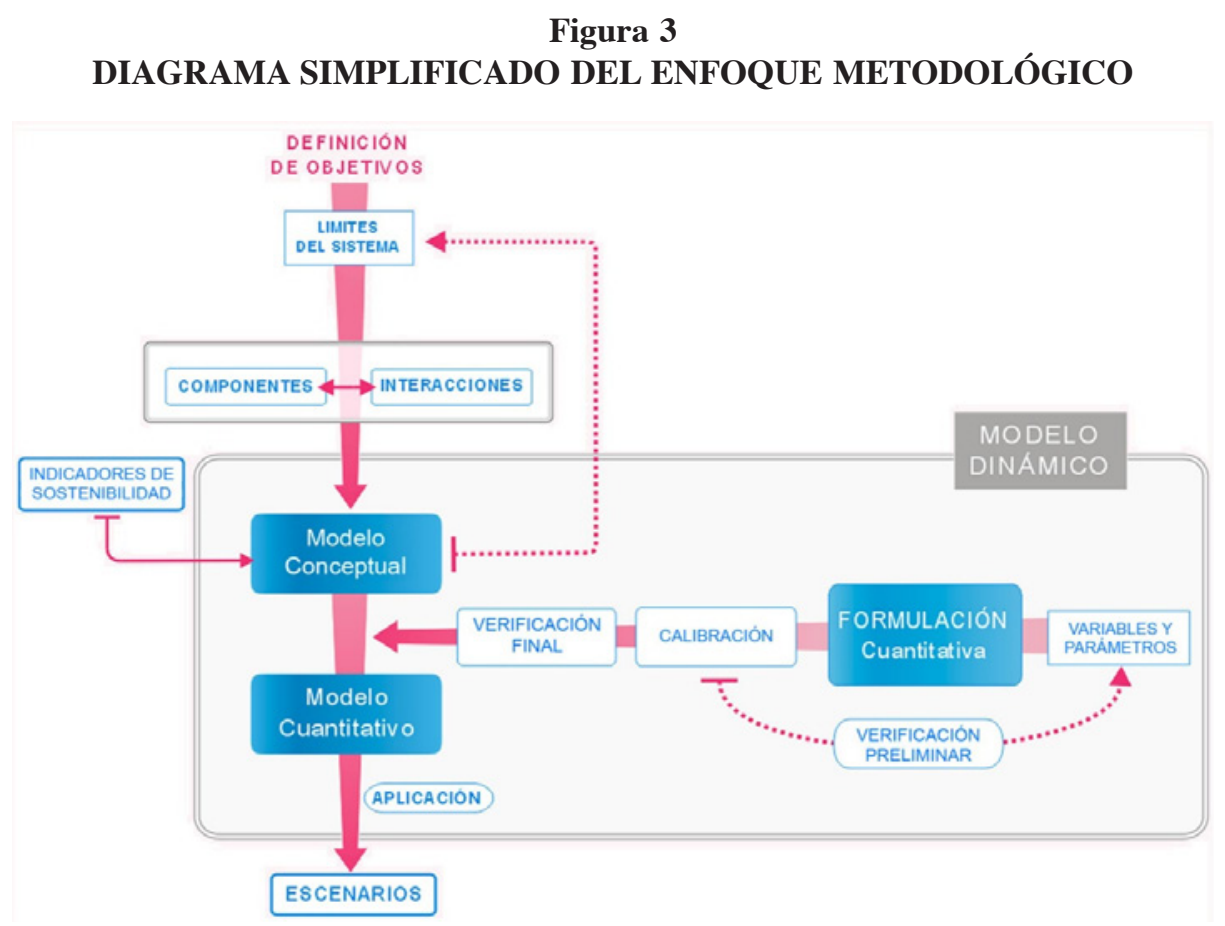

Este modelo cuantitativo se sometió a diversos test de verificación y calibración para el periodo 1996-2011 (Barlas, 1996), incluyendo: test de consistencia dimensional, análisis de sensibilidad, test de condiciones extremas y test de bondad de ajuste para las 20 variables con series históricas disponibles. Cuando se alcanza el grado de confianza requerido, el modelo se aplica en la exploración de comportamientos futuros bajo diferentes escenarios y políticas.

\subsection{Descripción del modelo}

El modelo global se estructura en 5 sectores (socio-turístico, usos del suelo, biodiversidad, recursos hídricos y calidad ambiental) e integra 37 indicadores de sostenibilidad ambiental y socioeconómica (ver detalles en Banos-González et al., 2015 y 2016). En este trabajo, nos hemos centrado en tres sectores del modelo, directamente relacionados con la dinámica socio-turística, que se detallan a continuación. 


\subsubsection{Sector socio-turístico}

Este bloque refleja la dinámica de la población residente y el turismo (Figura 4), considerados el motor de los cambios en los usos del suelo, así como de las demandas de recursos naturales, como el agua y la energía. Uno de los factores clave en este sector y también en el modelo general, es la población turística equivalente (PTE), definida como la población turística que equivale a la residente en función de la entrada anual de turistas y el número medio de pernoctaciones. Este indicador permite analizar la presión del turismo sobre el territorio y los recursos naturales, con independencia de la estacionalidad (BPIA, 2012). Su modelización se ha basado principalmente en el índice de elección turística, calculado en función de: i) la evolución del producto interior bruto de los principales mercados emisores de turistas hacia la isla (Garín-Muñoz, 2006), ii) la oferta de alojamiento turístico (Cruz, 2009) y iii) el índice de satisfacción turística (Santana-Jiménez y Hernández, 2011). Así mismo, se ha considerado el efecto de la Primavera Árabe sobre la llegada de turistas al mercado canario (Canalis, 2013). En cuanto a la dinámica de la población residente, destacar que los flujos migratorios están fuertemente influidos por la oferta de empleo turístico, que en Fuerteventura representa una media del $33 \%$ del empleo total en el momento del estudio (ISTAC, 2012), el cual depende a su vez de la PTE. La población residente y turística equivalente, así como la tasa de ocupación de los alojamientos turísticos existentes, va a determinar la demanda de nuevos alojamientos turísticos y residenciales que, a su vez, representa el principal motor de cambio en los usos del suelo y, en particular, en la transformación de la superficie con vegetación natural a coberturas artificiales (Santana-Jiménez et al., 2017).

\section{Figura 4 \\ DIAGRAMA DE FLUJO DEL SECTOR SOCIO-TURÍSTICO DEL MODELO DE SIMULACIÓN DINÁMICA DE LA RESERVA DE LA BIOSFERA DE FUERTEVENTURA}

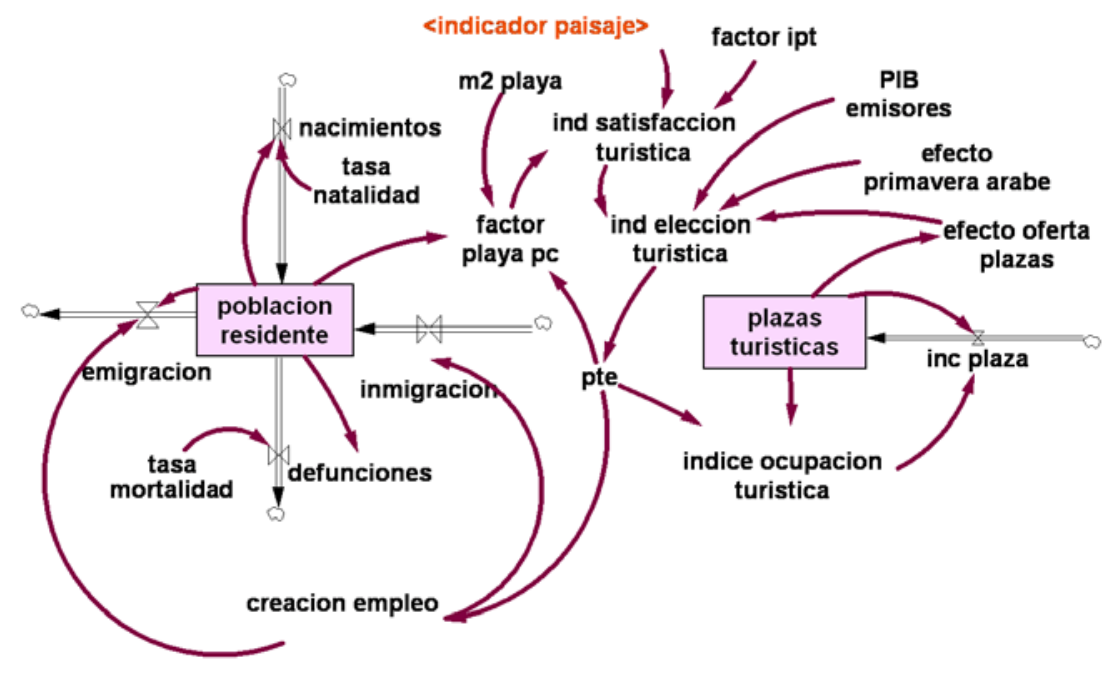

Cuadernos de Turismo, 43, (2019), 69-96 


\subsubsection{Sector de los recursos hídricos}

La escasez de recursos hídricos tradicionalmente ha representado uno de los factores limitantes para el desarrollo de la isla y, particularmente, para su desarrollo turístico y agrícola (Palacios et al., 2009). Los recursos superficiales son insuficientes para satisfacer las crecientes demandas de la población o los requisitos de riego. Los recursos de aguas subterráneas, predominantemente salobres (Herrera y Custodio, 2000), están destinados a usos agrícolas, debiendo desalinizarse antes de ser utilizados. Esto da una idea de la importancia del papel desempeñado por la desalación para cubrir la demanda total de agua (Cabrera y Custodio, 2012), así como la importancia del suministro de agua en la sostenibilidad de una isla turística hiperárida como ésta (Deyá y Tirado, 2011).

\subsubsection{Sector de calidad ambiental}

Esta parte del modelo tiene en cuenta las demandas de energía, las emisiones de $\mathrm{CO}_{2}$ y la generación de residuos. Incorpora las diferentes demandas de energía desde población turística equivalente, población residente, transporte, actividades productivas y la energía consumida por el proceso de desalinización. El aumento en el PIB regional conduce a un aumento en el índice de consumo eléctrico per cápita, así como en el índice de motorización, por lo que el modelo también permite calcular el indicador de autosuficiencia energética para analizar la proporción de la demanda total de energía cubierta por recursos renovables -una de las prioridades del Plan de Acción de la Reserva de la Biosfera-, que son principalmente: energía eólica, solar térmica y fotovoltaica (Denis y Parker, 2009; Chester, 2010). En cuanto a la gestión de los residuos urbanos, se consideraron la eficiencia de la separación y el reciclaje, y la cantidad de desechos tratados en vertederos. A través del índice de gestión selectiva de residuos se puede analizar la proporción de residuos urbanos reciclados, como otro de los componentes clave de la sostenibilidad de la isla (Cáceres, 2010).

\subsection{Evaluación de la reacción de los indicadores de sostenibilidad en diferentes escenarios y medidas políticas}

Con el fin de realizar una evaluación cuantitativa de la evolución de esta isla en términos de sostenibilidad, en este trabajo se ha analizado el comportamiento de 6 indicadores bajo diferentes escenarios y medidas gestión para el período 2012-2025. Dichos indicadores fueron seleccionados entre los 37 totales en función de sus vínculos con la dinámica socio-turística y con las políticas y escenarios involucrados; así como de la disponibilidad de los valores de sus umbrales, necesarios para evaluar su evolución en términos de sostenibilidad (Tabla 1). Siguiendo el criterio de varios autores (Gallopin, 1997; Moldan et al., 2012), la identificación de umbrales o valores de referencia es esencial a la hora de controlar las tendencias en términos de sostenibilidad en escenarios futuros, pudiendo aumentar la influencia real de los indicadores en la adopción de políticas sostenibles. 
Tabla 1

INDICADORES SELECCIONADOS, SU FORMULACIÓN EN EL MODELO E INFORMACIÓN SOBRE SUS RESPECTIVOS UMBRALES DE SOSTENIBILIDAD

\begin{tabular}{|c|c|c|c|}
\hline Indicadores & Formulación en el modelo & Variables involucradas & $\begin{array}{l}\text { Umbral de } \\
\text { sostenibilidad y su } \\
\text { significado } \\
\text { (Fuente que } \\
\text { establece dicho } \\
\text { umbral) } \\
\end{array}$ \\
\hline $\begin{array}{l}\text { Ratio entre los } \\
\text { alojamientos } \\
\text { turísticos y } \\
\text { la población } \\
\text { residente }(R A T P R)\end{array}$ & $R A T P R=\frac{c a t}{r e s}$ & $\begin{array}{l}\text { cat: número de plazas en } \\
\text { alojamientos turísticos. } \\
\text { res: población residente. }\end{array}$ & $\begin{array}{l}(<0,97) \text {. La } \\
\text { proporción de } \\
\text { alojamientos } \\
\text { turísticos y } \\
\text { población local debe } \\
\text { ser menor que } 0,97 \\
\text { camas turísticas / } \\
\text { habitante. } \\
\text { (Gobierno de } \\
\text { Canarias, 2008) } \\
\end{array}$ \\
\hline $\begin{array}{l}\text { Ratio turista- } \\
\text { residente } \\
(T U R E S)\end{array}$ & TURES $=\frac{\text { pte }}{\text { res }}$ & $\begin{array}{l}\text { pte: población turística } \\
\quad \text { equivalente. } \\
\text { res: población residente. }\end{array}$ & $\begin{array}{l}(<0,3152) . \mathrm{La} \\
\text { proporción de } \\
\text { turistas equivalente } \\
\text { y población local } \\
\text { debe ser menor } \\
\text { que }<0,3152 \\
\text { (Adimensional). } \\
\text { (Gobierno de } \\
\text { Canarias, 2008) }\end{array}$ \\
\hline $\begin{array}{l}\text { Proporción } \\
\text { de superficie } \\
\text { artificial }(P S A)\end{array}$ & $P S A=\frac{r e a+h o t+n h o+g o f+r o d+t r a+i r r}{F v a}$ & $\begin{array}{l}\text { rea: } \text { área ocupada por usos } \\
\text { residenciales. } \\
\text { hot: } \text { área ocupada } \\
\text { por hoteles y sus } \\
\text { instalaciones. } \\
\text { nho: área ocupada por } \\
\text { servicios de alojamiento } \\
\text { no hoteleros y sus } \\
\text { instalaciones. } \\
\text { gof: área ocupada por } \\
\text { campos de golf. } \\
\text { rod: área ocupada por } \\
\text { carreteras. } \\
\text { tra: } \text { área ocupada por } \\
\text { pistas o caminos no } \\
\text { pavimentados. } \\
\text { irr: área ocupada por } \\
\text { cultivos de riego. } \\
\text { Fva: Área de la isla de } \\
\text { Fuerteventura. }\end{array}$ & $\begin{array}{l}(<20) \text {. El } \\
\text { porcentaje de } \\
\text { superficie artificial, } \\
\text { ocupada por usos } \\
\text { agrícolas, urbanos } \\
\text { e infraestructuras, } \\
\text { respecto al total debe } \\
\text { ser menor del } 20 \% \\
\text { (Graymore } \text { et al., } \\
2010)\end{array}$ \\
\hline
\end{tabular}




\begin{tabular}{|c|c|c|c|}
\hline Indicadores & Formulación en el modelo & Variables involucradas & \begin{tabular}{|l} 
Umbral de \\
sostenibilidad y su \\
significado \\
(Fuente que \\
establece dicho \\
umbral) \\
\end{tabular} \\
\hline $\begin{array}{l}\text { Energía Primaria } \\
\text { utilizada per capita } \\
(\mathrm{EPpc})\end{array}$ & $E P_{p c}=\frac{p e p+p e i+p e d+p e v+p e n}{t p o}$ & $\begin{array}{l}\text { pep: energía primaria urbana } \\
\text { consumida. } \\
\text { pei: energía primaria } \\
\text { consumida en el sector } \\
\text { industrial. } \\
\text { ped: energía primaria } \\
\text { consumida en } \\
\text { los procesos de } \\
\text { desalinización. } \\
\text { pev: energía primaria } \\
\text { consumida por } \\
\text { vehículos en la isla. } \\
\text { pen: energía primaria } \\
\text { consumida en el sector } \\
\text { de transporte (barcos y } \\
\text { vuelos). } \\
\text { tpo: población total. }\end{array}$ & $\begin{array}{l}(<42) \text {. La energía } \\
\text { mínima requerida } \\
\text { para alcanzar un } \\
\text { Índice de Desarrollo } \\
\text { Humano de al } \\
\text { menos } 0,8, \text { según lo } \\
\text { recomendado por } \\
\text { el PNUD, no debe } \\
\text { superar los } 42 \text { GJ/ } \\
\text { año*pc. } \\
\text { (Johansson y } \\
\text { Goldemberg, 2008) }\end{array}$ \\
\hline $\begin{array}{l}\text { Emisiones de } \mathrm{CO}_{2} \\
\text { per capita }\left(\mathrm{CO}_{2} \mathrm{pc}\right)\end{array}$ & $\mathrm{CO}_{2} \mathrm{pc}=\frac{c_{\text {elc }}+c_{\text {wst }}+c_{\text {nav }}+c_{\text {veh }}+c_{\text {gav }}+c_{i r r}+c_{g o f}}{\text { tpo }}$ & $\begin{array}{l}\text { celc: Emisiones de } \mathrm{CO}_{2} \\
\text { del consumo de } \\
\text { electricidad. } \\
\text { cwst: Emisiones de } \mathrm{CO}_{2} \\
\text { de la producción de } \\
\text { residuos urbanos. } \\
\text { cnav: Emisiones de } \mathrm{CO}_{2} \text { del } \\
\text { sector de transportes } \\
\text { (barcos y vuelos). } \\
\text { cveh: Emisiones de } \mathrm{CO}_{2} \text { de } \\
\text { los vehículos en la isla. } \\
\text { cgav: Emisiones de } \mathrm{CO}_{2} \text { de } \\
\text { gavias activas. } \\
\text { cirr: Emisiones de } \mathrm{CO}_{2} \text { del } \\
\text { cultivo de riego. } \\
\text { cgof: Emisiones de } \mathrm{CO}_{2} \text { del } \\
\text { área de campos de golf. } \\
\text { tpo: población total. } \\
\end{array}$ & $\begin{array}{l}(<9,52) \text {. Las } \\
\text { emisiones de } \\
\mathrm{CO}_{2} \text { per cápita } \\
\text { no deben superar } \\
\text { las } 9,52 \text { toneladas } \\
\text { métricas } \mathrm{CO}_{2} / \text { año } \\
* \text { pc. De este modo, } \\
\text { se conseguiría una } \\
\text { reducción del } 20 \% \\
\text { en las emisiones de } \\
\mathrm{CO}_{2} \text { per cápita desde } \\
\text { los niveles de } 1990 . \\
\text { (EC, 2008) }\end{array}$ \\
\hline $\begin{array}{l}\text { Índice de } \\
\text { autosuficiencia } \\
\text { energética (IAE) }\end{array}$ & $I A E=\frac{w p+t h p+p h p}{p e p+p e i+p e d}$ & $\begin{array}{l}w p, \text { thp and } p h p \text { son la } \\
\text { energía producida por } \\
\text { recursos renovables: } \\
\text { energía eólica, } \\
\text { energía térmica y } \\
\text { energía fotovoltaica, } \\
\text { respectivamente. } \\
\text { pep, pei and ped son la } \\
\text { demanda de energía } \\
\text { primaria de la población } \\
\text { (residente y turística), } \\
\text { las actividades } \\
\text { productivas y la } \\
\text { desalación de agua de } \\
\text { mar, respectivamente. }\end{array}$ & $\begin{array}{l}(>0,2) \text {. Una cuota de } \\
\text { energía renovable } \\
\text { de al menos } 20 \% \text { en } \\
2020 \text { y } 27 \% \text { en } 2030 \\
\text { (Adimensional). } \\
\text { EC }(2008,2015)\end{array}$ \\
\hline
\end{tabular}




\subsection{Descripción de los escenarios y tomas de decisión políticas}

Los sistemas de análisis empleados en la toma de decisiones para la mejora de la gestión de los recursos, deben permitir evaluar la capacidad de resiliencia de un sistema, es decir, hacer frente a los cambios, tanto internos como externos. Con el fin de incorporar una evaluación preliminar de esta capacidad en nuestro análisis, se exploraron para el periodo 2012-2025 dos escenarios climáticos, dos escenarios económicos y dos medidas de gestión política.

\subsubsection{Escenarios de cambio climático}

Para determinar el efecto del calentamiento global, se consideraron los escenarios A2 y B2 del informe especial en escenarios de emisión del Grupo Intergubernamental de Expertos sobre el Cambio Climático (IPCC, 2014). En el MSF, los parámetros del modelo para los escenarios A2 y B2, fueron calculados sobre la base del informe CEDEX (2011). Estos escenarios implican una disminución del $10 \%$ en la lluvia anual y un aumento del $13 \%$ en los requisitos de riego en 2025 para A2 y una disminución de la precipitación anual del 14\% y el aumento de las necesidades de riego del $18 \%$ para el escenario B2.

\subsubsection{Escenarios económicos.}

Se consideraron dos escenarios económicos: el primero es el de Crecimiento, definido por una serie de variables macroeconómicas (PIB canario, el PIB medio de los principales países emisores de turistas para Fuerteventura, y el índice de precios turísticos), que muestran un comportamiento promedio similar a la tendencia histórica del periodo 1996-2007. Este escenario representa un período de notable crecimiento, alrededor de 2,65 veces mayor que el comportamiento promedio bajo un escenario tendencial o 'sin cambios' (ESC), periodo en el que varios autores (Alcamo et al., 2007; Qin et al., 2011; Schaldach et al., 2012; Matos et al., 2014) señalan la importancia del aumento en el consumo de recursos naturales, como el agua y la energía. El segundo escenario se basó en un periodo de Recesión, caracterizado por unas variables macroeconómicas que muestran una recesión económica, con un comportamiento promedio similar al observado en el período 2008-2011.

\subsubsection{Medidas de gestión política}

Se han implementado en el modelo dos medidas. Por un lado, la Medida I (M.I), que consistiría en cubrir el $100 \%$ de la demanda de electricidad para el suministro de agua desalinizada con energía renovable. Esta medida representa uno de los planteamientos esenciales en la Reserva de la Biosfera (AP, 2013 y comunicación personal). Y la Medida II (M.II), que consistiría en la limitación de la construcción de nuevos alojamientos turísticos, y se implementaría en el modelo aumentando el umbral de la tasa de ocupación de los alojamientos turísticos existentes al 75\%, en línea con los informes sobre capacidad de carga en zonas turísticas de la Consejería de Medio Ambiente y Ordenación Territorial 
(Gobierno de Canarias, 2008). En trabajos previos (Banos-González, 2016), se demostró la mayor eficacia de esta medida basada en la mejora de los índices de ocupación de los alojamientos turísticos existentes en un destino como Fuerteventura, frente a medidas basadas únicamente en el establecimiento de un número máximo de plazas alojativas, en línea con lo establecido por el Gobierno de Canarias (2003). Cabe recordar en este punto, que esta tasa de ocupación determina, junto a otras variables del modelo, la construcción de nuevas plazas turísticas.

\section{RESULTADOS Y DISCUSIÓN}

Este apartado se estructura en tres bloques. En el primero de ellos, se describen los resultados de los test de verificación y calibración. Una vez probada la confianza en el modelo, se aplica el modelo para el análisis de la evolución de los factores clave de la sostenibilidad y sus principales interacciones en la isla. Finalmente, se explora el comportamiento de determinados indicadores de sostenibilidad integrados en el modelo bajo distintos escenarios y medidas de gestión, lo que permite realizar una reflexión sobre el potencial de esta herramienta para realizar un análisis integral del funcionamiento y evolución de sistemas socioecológicos vulnerables, como es el caso de la isla de Fuerteventura.

\subsection{Resultados de los test de verificación}

El modelo superó satisfactoriamente los distintos test de verificación aplicados. El análisis de sensibilidad, necesario para evaluar la robustez del modelo (Graham et al., 2002), se llevó a cabo aplicando un análisis de sensibilidad local y una simulación de Monte Carlo. Estos análisis mostraron una sensibilidad entre baja y moderada del modelo a los cambios en los valores de los parámetros, lo que permite considerar que el modelo es robusto en sus resultados, es decir, que no dependen de forma crítica de posibles variaciones en tales parámetros. En relación con las pruebas de condiciones extremas (Li et al., 2012), el modelo generó los resultados esperados cuando se implementaron un total de 25 condiciones extremas diferentes, como una caída inesperada de las llegadas de turistas, una demanda acelerada de la construcción o sequías extremas.

La comparación de los resultados de la simulación con los datos observados constituye una medida de la bondad del ajuste y, por lo tanto, de la capacidad del modelo para rastrear el comportamiento real del sistema (Martínez-Moyano y Richardson, 2013). En este trabajo se han determinado las evaluaciones del porcentaje del error absoluto medio (MAPE), calculado según Goh y Law (2002) y Oliva (2003); y la raíz del error cuadrático medio normalizado (NRMSE), según Andarizan et al. (2011) y Sepaskhah et al. (2013) para las 20 variables con series observadas disponibles. Los resultados de las 20 variables muestran valores similares para ambas estadísticas (Tabla 2).

Un total de 14 variables (Tabla 2) tienen un porcentaje de error absoluto por debajo del $10 \%$, que se considera un excelente grado de ajuste (Goh y Law 2002), mientras que 4 variables $(14,15,16$ y 18) logran un buen grado de ajuste (MAPE entre 10-20\%). Solo dos variables, inmigración y emigración, tienen un grado de ajuste sólo aceptable (MAPE entre 20-30\%), Respecto al cálculo del NRMSE, 12 variables presentan un excelente grado 
de ajuste según Andarizan et al. (2011) y Sepaskhah et al. (2013), 5 variables logran un buen grado de ajuste, y 3 variables logran un grado aceptable: inmigración, emigración y campos de golf.

Tabla 2

EVALUACIONES DEL PORCENTAJE DEL ERROR ABSOLUTO MEDIO (MAPE) Y RAÍZ DEL ERROR CUADRÁTICO MEDIO NORMALIZADO (NRMSE) DE LA COMPARACIÓN DE LOS RESULTADOS DE LA SIMULACIÓN CON LOS DATOS OBSERVADOS PARA 20 VARIABLES CON SERIES DISPONIBLES

\begin{tabular}{clccc}
\hline \multirow{2}{*}{ Identificador } & \multicolumn{1}{c}{ Variables } & n & $\begin{array}{c}\text { Resultados para el periodo de } \\
\text { calibración }\end{array}$ \\
& & & MAPE (\%) & RMSE (\%) \\
\hline 1 & Población residente & 16 & 4,30 & 5,45 \\
2 & Nacimientos & 12 & 6,22 & 5,62 \\
3 & Inmigración & 16 & 26,18 & 23,38 \\
4 & Emigración & 15 & 32,70 & 31,65 \\
5 & Población turística equivalente & 16 & 9,52 & 12,03 \\
6 & Capacidad de alojamiento turístico & 16 & 7,29 & 9,4 \\
7 & Tasa de ocupación & 16 & 8,71 & 10,84 \\
8 & Empleo turístico & 13 & 5,39 & 6,63 \\
9 & Hábitat de la hubara & 3 & 0,98 & 1,53 \\
10 & Población de guirre real & 13 & 4,54 & 5,08 \\
11 & Urbano edificado & 16 & 2,34 & 2,84 \\
12 & Caminos & 3 & 1,06 & 1,73 \\
13 & Carreteras & 3 & 0,71 & 1,05 \\
14 & Área de cultivos & 15 & 10,14 & 11,40 \\
15 & Área de cultivos de regadío & 15 & 11,76 & 13,70 \\
16 & Área de gavias activas & 15 & 10,49 & 11,55 \\
17 & Área de vegetación natural & 3 & 0,28 & 0,45 \\
18 & Área de campos de golf & 15 & 10,01 & 24,45 \\
19 & Flota de vehículos & 12 & 4,57 & 4,15 \\
20 & Consumo de energía eléctrica & 14 & 4,98 & 7,14 \\
\hline
\end{tabular}

n: Número de datos observados.

En conjunto, estos resultados de calibración y verificación ofrecen un elevado grado de confianza en el modelo para su aplicación en el análisis de la evolución de los factores clave de la sostenibilidad y sus principales interacciones en la isla (Ver Banos-González et al. 2015 y 2016 para más detalles). El MSF permite, por tanto, el análisis de las principales interacciones entre los procesos demográficos y socioeconómicos, así como su influencia en el binomio agua-energía. 


\subsection{Principales resultados de la simulación para el periodo de calibración}

Durante el periodo de calibración 1996-2011, el modelo refleja adecuadamente la tendencia creciente de la población turística equivalente, así como el efecto de la crisis económica, que dio comienzo en 2007 con una gran repercusión en el factor PIB, y que provocó una marcada caída de los turistas llegados en 2009 (Figura 5a). Diversos factores como el inicio de la recuperación económica en los principales países emisores de turistas hacia Fuerteventura y la contracción de los precios turísticos en la isla en los años 2009 y 2010, favorecieron la recuperación de la PTE a partir de entonces.

\section{Figura 5}

\section{RESULTADOS DE LOS DATOS OBSERVADOS Y RESULTADOS DE SIMULACIÓN ENTRE 1996 Y 2011. A) POBLACIÓN TURÍSTICA EQUIVALENTE. B) EMPLEO TURÍSTICO. C) POBLACIÓN RESIDENTE. D) ALOJAMIENTOS TURÍSTICOS}

a)

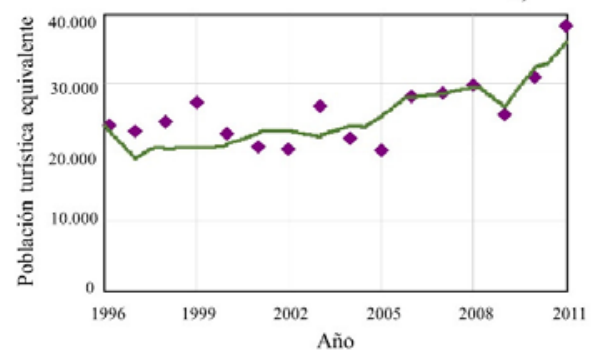

c)

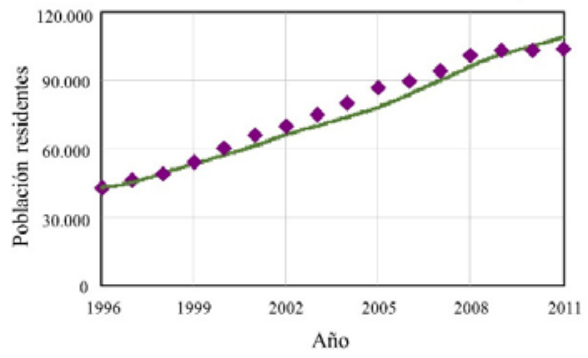

Datos de observación

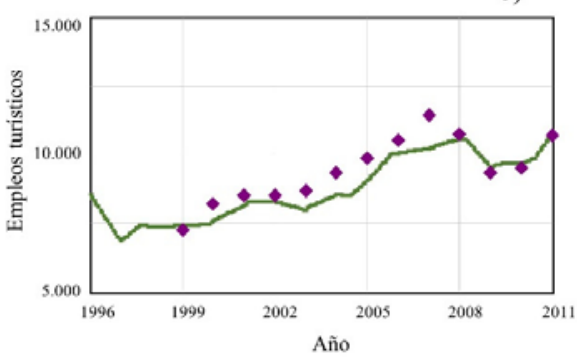

d)

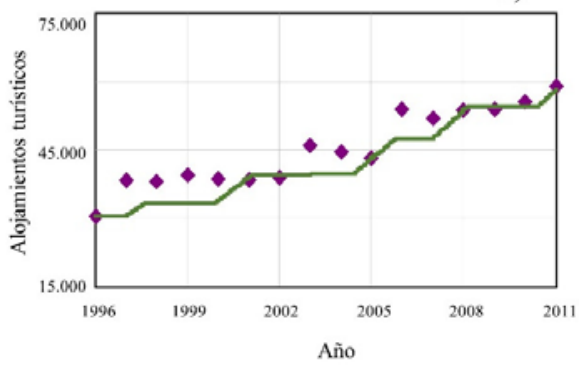

Resultados de la simulación

Fuente de datos de observación: ISTAC (2010).

La actividad turística es uno de los factores clave en el extraordinario crecimiento demográfico acontecido en Fuerteventura, habiéndose duplicado la población residente en sólo 10 años (1996-2006), superando los 100.000 residentes al final del periodo de calibración (Figura 5c). Esta evolución no sólo se explica por un fuerte crecimiento vegetativo (Figura 6a), sino que se debe principalmente a un saldo migratorio positivo motivado 
por el poder de atracción poblacional de la actividad turística y de las diversas ramas productivas que es capaz de estimular (Figura 5b) (Santana-Jiménez y Hernández, 2011).

En cuanto a los recursos hídricos, hay una notable falta de datos observados. Sin embargo, los valores y los rangos de los resultados de la simulación son consistentes con la información disponible. El aumento en la población residente y equivalente de turistas representa un factor determinante en el consumo de recursos naturales, como la energía y el agua. El consumo de agua neto por residente durante el periodo de calibración fue de 180 litros por persona y por día (Figura 6c); mientras que los turistas consumieron alrededor de 378 litros y 221 litros por persona y día en hoteles y alojamientos turísticos no hoteleros, respectivamente (CIAFV, 2009). La demanda de campos de golf, regadío y demanda de ganado corresponde a alrededor de 2,31, 3,45 y 0,23 hm³/año, respectivamente, al final del período de simulación. A la luz de los datos disponibles, los recursos superficiales y subterráneos son claramente insuficiente para satisfacer la demanda total de agua, llegando a cubrir entorno al $20 \%$ de la misma, siendo la desalación de agua de mar la que ha permitido abordar las limitaciones de la escasez de agua en las actividades socioeconómicas.

\section{Figura 6 \\ DATOS OBSERVADOS Y RESULTADOS DE LA SIMULACIÓN ENTRE 1996 Y 2011. A) NACIMIENTOS. B) RATIO DE OCUPACIÓN. C) DEMANDA DE AGUA. D) CONSUMO ELÉCTRICO}

a)

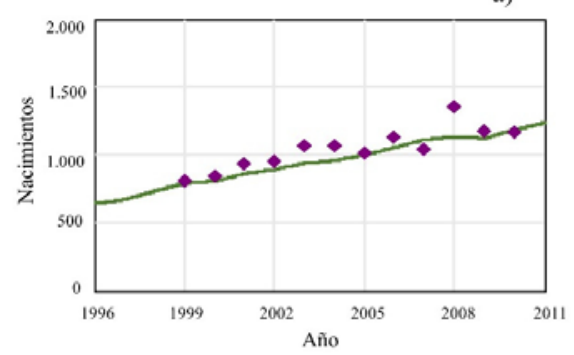

c)

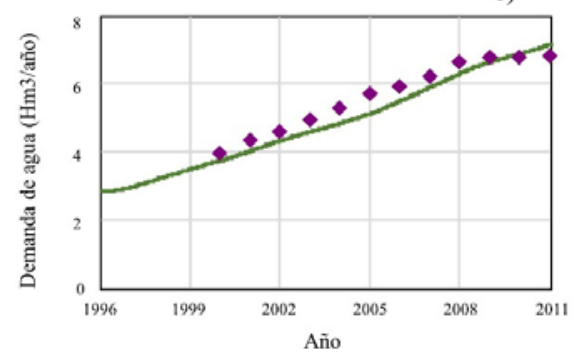

Datos de observación

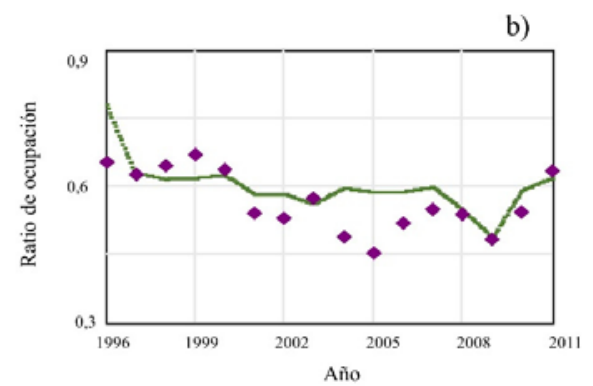

d)

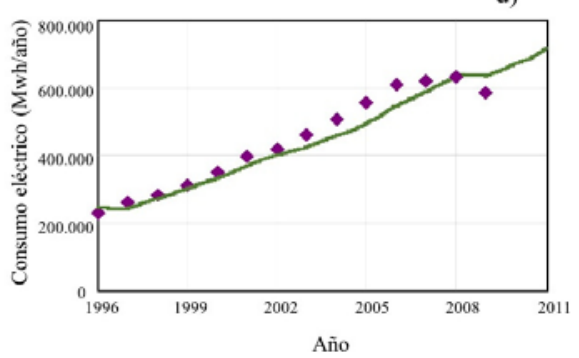

Resultados de la simulación

Fuente de datos de observación: ISTAC (2010). 
Como cabía esperar, el consumo total de energía eléctrica se ha incrementado durante el periodo de calibración (Figura 6d), debido al aumento del consumo eléctrico per cápita, relacionado con la mejora general del PIB regional y con el incremento de la población total (residente y turístico equivalente) así como en relación a la demanda de agua para abastecimiento urbano. Sin embargo, la fuerte dependencia del consumo de energía para el suministro de agua, dado que el $80 \%$ de la demanda total de agua se obtiene por la desalación de agua de mar, implica una alta vulnerabilidad de todo el sistema socioecológico frente a los cambios socioeconómicos relacionados con los mercados energéticos, incluso para cubrir las necesidades hídricas básicas (Kruyt et al., 2009). De hecho, uno de los objetivos del Plan de Acción de la Reserva de la Biosfera de Fuerteventura (AP, 2013) es, precisamente, la reducción de la dependencia energética de fuentes de energía alóctonas y no renovables, con el fin de reducir esa vulnerabilidad en el futuro próximo y, sobre todo, ante escenarios de cambio global.

\subsection{Comportamiento de los indicadores de sostenibilidad bajo distintos escenarios y medidas de gestión}

De acuerdo con Folke et al. (2005), una herramienta real para la gestión sostenible en sistemas socioecológicos debería tener la capacidad de evaluar los efectos de los cambios en los factores externos sobre el sistema objeto de estudio. Con el fin de analizar la vulnerabilidad de la isla de Fuerteventura a este tipo de cambios, el modelo se ha aplicado para evaluar el comportamiento del sistema bajo distintos escenarios y medidas de gestión para el periodo 2012-2025, utilizando el conjunto de indicadores y sus umbrales recogidos en la Tabla 1.

La Tabla 3 muestra los resultados en 2025 de estos indicadores bajo la simulación del escenario tendencial (ESC), los dos escenarios climáticos, los dos escenarios económicos y las dos medidas de gestión.

\section{Tabla 3}

\section{RESULTADOS DE LAS SIMULACIONES DE LOS INDICADORES DE SOSTENIBILIDAD, BAJO LOS DISTINTOS ESCENARIOS Y MEDIDAS (VALORES EN 2025)}

\begin{tabular}{|c|c|c|c|c|c|c|c|c|}
\hline \multirow[t]{2}{*}{ Indicadores } & \multirow[t]{2}{*}{ Umbrales } & \multirow[t]{2}{*}{ ESC } & \multicolumn{2}{|c|}{$\begin{array}{c}\text { Escenarios de } \\
\text { cambio climático }\end{array}$} & \multicolumn{2}{|c|}{ Escenarios económicos } & \multicolumn{2}{|c|}{$\begin{array}{l}\text { Medidas } \\
\text { políticas }\end{array}$} \\
\hline & & & A2 & B2 & Crecimiento & Recesión & M.I & M.II \\
\hline $\begin{array}{l}\text { Ratio entre los } \\
\text { alojamientos } \\
\text { turísticos y la } \\
\text { población residente } \\
(R A T P R)\end{array}$ & $<0,97$ & 0,46 & 0,46 & 0,46 & 0,411 & 0,489 & 0,46 & 0,42 \\
\hline $\begin{array}{l}\text { Ratio turista- } \\
\text { residente (TURES) }\end{array}$ & $<0,3152$ & 0,329 & 0,329 & 0,329 & 0,312 & 0,339 & 0,329 & 0,426 \\
\hline $\begin{array}{l}\text { Proporción de } \\
\text { superficie artificial } \\
(P S A)\end{array}$ & $<20$ & 9,78 & 9,78 & 9,78 & 12,09 & 9,48 & 9,78 & 7,55 \\
\hline $\begin{array}{l}\text { Energía Primaria } \\
\text { utilizada per cápita } \\
(\mathrm{EPpc})\end{array}$ & $<42$ & 262,73 & 262,83 & 262,96 & 297,29 & 253,66 & 256,46 & 283,01 \\
\hline
\end{tabular}




\begin{tabular}{|c|c|c|c|c|c|c|c|c|}
\hline \multirow{2}{*}{ Indicadores } & \multirow{2}{*}{ Umbrales } & \multirow{2}{*}{ ESC } & \multicolumn{2}{|c|}{$\begin{array}{c}\text { Escenarios de } \\
\text { cambio climático }\end{array}$} & \multicolumn{2}{|c|}{ Escenarios económicos } & \multicolumn{2}{|c|}{$\begin{array}{l}\text { Medidas } \\
\text { políticas }\end{array}$} \\
\hline & & & A2 & B2 & Crecimiento & Recesión & M.I & M.II \\
\hline $\begin{array}{l}\text { Emisiones de } \mathrm{CO}_{2} \\
\text { per cápita }\left(\mathrm{CO}_{2} \mathrm{pc}\right)\end{array}$ & $<9,52$ & 15,98 & 16,11 & 16,16 & 17,34 & 15,76 & 15,82 & 17,63 \\
\hline $\begin{array}{l}\text { Índice de } \\
\text { autosuficiencia } \\
\text { energética (IAE) }\end{array}$ & $>0,2$ & 0,010 & 0,010 & 0,010 & 0,008 & 0,0113 & 0,02 & 0,01 \\
\hline
\end{tabular}

Figura 7

RESULTADOS DE SIMULACIÓN BAJO EL ESCENARIO TENDENCIAL (ESC), BAJO LOS ESCENARIOS DE CAMBIO CLIMÁTICO (A2; B2), BAJO LOS ESCENARIOS ECONÓMICOS (CRECIMIENTO; RECESIÓN), Y BAJO LAS DOS MEDIDAS DE POLÍTICA (M.I; M.II) PARA LOS INDICADORES DE SOSTENIBILIDAD SELECCIONADOS. LOS ESCENARIOS A2 Y B2 NO VARÍAN EN LAS SIMULACIONES B), C), D), E) Y F), COINCIDIENDO CON EL ESCENARIO TENDENCIAL. EL ESCENARIO M.I NO VARÍA EN LAS SIMULACIONES D), E) Y F), COINCIDIENDO CON EL ESCENARIO TENDENCIAL

a)
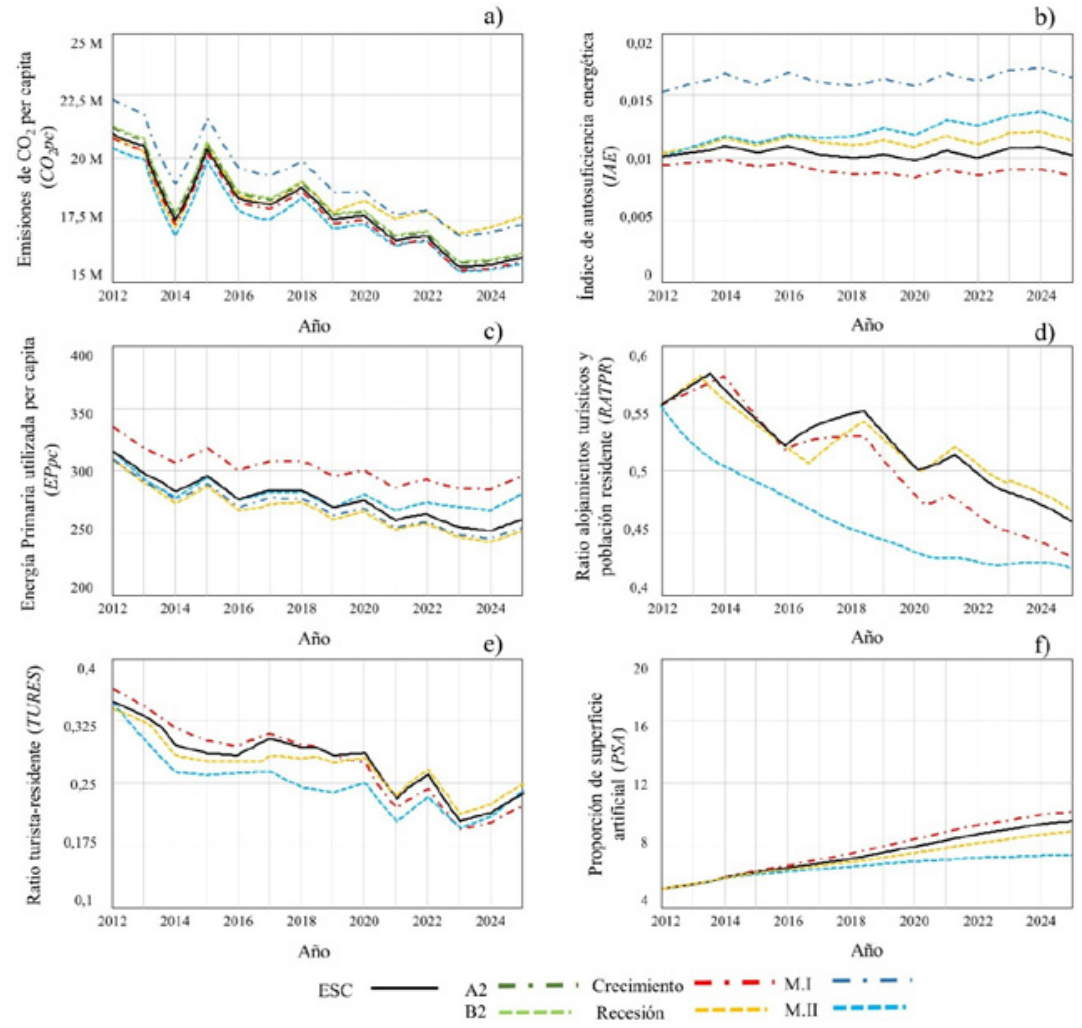

Cuadernos de Turismo, 43, (2019), 69-96 
La proporción entre turistas y población local (TURES) permite una aproximación a la evaluación de la presión que ejerce el turismo. De acuerdo con BPIA (2012), una alta proporción de turistas extranjeros por habitante conlleva una mayor necesidad de recursos y una mayor prestación de servicios, pudiendo derivar en un aumento de las presiones y de los impactos con consecuencias en la degradación del medio. A su vez, la relación turistas-residentes es útil para medir la calidad de vida de éstos últimos. La simulación tendencial del MSF muestra que, en 2025, este indicador podría exceder su umbral de sostenibilidad, sobre todo en los principales municipios turísticos, Pájara y La Oliva. Como se comentaba anteriormente, la puesta en marcha de la M.II, conduciría a una reducción de la población turística equivalente y la población residente en torno al $13 \%$ y $27 \%$ en comparación con ESC, respectivamente. Esta mayor disminución en los residentes llevaría a un empeoramiento de alrededor del 29,5\% de este indicador en M.II. En esta línea se esperaría la evolución de este indicador bajo un escenario de recesión económica, por la mayor caída de la población residente, frente a los turistas equivalentes. Parece que la dinámica de la variable población residente es más sensible a los cambios económicos que los turistas equivalentes, pues, bajo un escenario de bonanza económica, se espera un crecimiento mayor en esta variable y, por tanto, una mejora del indicador entorno a un $3 \%$ respecto a ESC.

La población turística equivalente y la residente representan los principales motores de cambio de otros indicadores de sostenibilidad territorial, como la proporción de superficie artificial (Spilanis et al., 2009; BPIA, 2012). La transformación del territorio a suelos urbanos y otros usos artificiales constituye uno de los cambios de uso que puede inducir en mayor medida procesos de insostenibilidad, pese a los aparentemente modestos valores de ocupación aportados respecto al territorio total. La proporción de superficie artificial (PSA) casi se duplicaría entre 2012 y 2025 bajo el escenario tendencial, y empeoraría entorno a un $24 \%$ bajo el escenario de crecimiento económico (Figura $7 f$ ). Por el contrario, la moderación en la construcción de nuevas infraestructuras contemplada bajo un escenario de contención económica, podría mejorar este indicador alrededor del 3\%. En el caso de la implantación de la M.II, PSA mejoría, ya que se espera que esta medida ralentice de manera notable los procesos de urbanización. Sin embargo, en cualquiera de las simulaciones, este indicador se mantendría lejos de su umbral de sostenibilidad, como cabe esperar de una isla como Fuerteventura, donde el desarrollo urbano-turístico fue más tardío en comparación con otras islas del archipiélago canario, como Tenerife y Gran Canaria, donde el desarrollo urbanístico se inició décadas anteriores.

Se observa en todas las simulaciones analizadas que, tanto RATPR como PSA, permanecen lejos de sus umbrales, lo que podría significar un buen resultado en términos de sostenibilidad. Esto podría interpretarse como una posibilidad para dar respuesta a una creciente demanda de infraestructuras turísticas y residenciales en la isla. Sin embargo, es complicado mantener un aumento moderado de la llegada de turistas, favorecer la conservación de algunos de sus atractivos naturales y, al mismo tiempo, sustentar un equilibrio entre la población local y otros agentes sociales interesados (Dodds, 2007; SantanaJiménez y Hernández, 2011). Por tanto, en línea con los objetivos del Plan de Acción de la Reserva de la Biosfera de Fuerteventura, sería adecuado evitar el crecimiento descontrolado, ya incipiente en Fuerteventura y experimentado por otras islas del archipiélago. 
En relación a estos efectos, los resultados del modelo en el periodo 2012-2025 respaldan el mantenimiento de ciertas restricciones en la construcción de nuevos establecimientos turísticos, apostando por un mayor aprovechamiento y rehabilitación de los existentes. Así mismo, ponen de manifiesto la eficacia de la Medida II para alcanzar esos objetivos en una isla como Fuerteventura, en línea con lo sugerido por otros autores (Oreja-Rodríguez et al., 2008; Santana Jiménez y Hernández, 2011).

En cuanto a los indicadores del sector de calidad ambiental, muy influidos por la dinámica socio-turística, los resultados muestran que, bajo el ESC, el consumo total de energía primaria y las emisiones totales de $\mathrm{CO}_{2}$ aumentarían en Fuerteventura alrededor de $36 \%$ y $25 \%$, respectivamente, entre 2012 y 2025 . Sin embargo, los indicadores per cápita, CO2pc y EPpc, mejorarían en ese periodo (Figuras 7 a y 7c, respectivamente). Durante el mismo, el aumento esperado de la participación de la producción de energía renovable (IAE) en la isla sería de alrededor del $1 \%$ bajo ESC.

A la luz de los resultados de la simulación, parece que, en 2025, el impacto del cambio climático sobre los indicadores estudiados sería aún limitado. En comparación con ESC, se esperarían ligeros aumentos en EPpc y $C O 2 p c$, siendo, para este último de $0,8 \%$ y $1,1 \%$ bajo A2 y B2, respectivamente, aunque sí apuntan a una tendencia al empeoramiento. La tendencia a corto y medio plazo hacia un periodo con menos precipitaciones, exigirá un aumento en el consumo de agua, tanto para usos urbanos como para el sector agrícola y ganadero, lo que impulsará el consumo energético, así como una probable pérdida en la capacidad de captura de $\mathrm{CO}_{2}$ de los sistemas naturales, que también es reflejado en la simulación del modelo.

Bajo un escenario de bonanza económica, tanto EPpc como CO2pc tenderían a empeorar respecto a la simulación tendencial, dada la tendencia a aumentar el consumo de recursos naturales, como el agua y la energía. Cuando las circunstancias económicas empeoran, el consumo tiende a contraerse, y ello podría explicar la mejora de estos indicadores bajo este escenario.

Bajo M.I, en el que la demanda de energía de los procesos de desalinización se abastecería con fuentes renovables, el EPpc y el CO2pc caerían alrededor de un 2,4\% y un $1 \%$, respectivamente, en relación con el escenario tendencial. El IAE casi se duplicaría (Figura $7 b$ ), aunque su impacto en el sistema total de energía sería aún bastante pequeño. Bajo la simulación de la M.II, se esperarían aumentos en estos indicadores, relacionados con la reducción de la población total, tanto turistas equivalentes como residentes.

Estos tres indicadores, $E P p c, C O 2 p c$ e $I A E$, pueden resultar útiles para cuantificar ciertos objetivos de sostenibilidad energética que recoge el Plan de Acción de la Reserva (AP, 2013), como la reducción de la dependencia de fuentes de energía alóctonas y no renovables; así como en cuanto a los objetivos generales establecidos en relación con las políticas europeas sobre energía y cambio climático (EC, 2015). Bajo todas las simulaciones, estos indicadores están lejos de cumplir los umbrales de sostenibilidad (Tabla 3), incluso bajo la medida I, que aborda explícitamente su mejora. Si bien M.I arrojaría mejores resultados que ESC para estos indicadores, parece insuficiente para cumplir dichos objetivos. Se requieren medidas y políticas más ambiciosas, especialmente en sistemas insulares, donde la provisión de energía a partir de combustibles fósiles implica una serie de dificultades, como los costos económicos y ambientales del uso de combustibles 
fósiles alóctonos y la dependencia del transporte del combustible en sí (Becken et al., 2003; Kuo y Chen, 2009). Algunos trabajos recogen los resultados de ciertas medidas en esta dirección implementadas en otras islas como la vecina El Hierro (Iglesias y Carvallo 2011), el Dodecaneso griego (Oikonomou et al., 2009) o la isla danesa Samsø (Nielsen y Jørgensen, 2015).

Finalmente, cabe reflexionar sobre la mejora que experimentan entre los años 2012 y 2025 ciertos indicadores relativos o per cápita, a pesar de los aumentos en el consumo de recursos y emisiones en términos absolutos. Esto se debe al aumento aún mayor en la población total a lo largo de ese período. Obviamente, esto no implica que si llegan más turistas a la isla, será más sostenible la Reserva de la Biosfera de Fuerteventura. Parece que algunos indicadores relativos, particularmente muchos indicadores de eficiencia, no siempre brindan información sólida sobre la sostenibilidad, cuando tales indicadores relativos se consideran de manera aislada y con valores discretos. Por tanto, estos indicadores de eficiencia y sus cambios a lo largo del tiempo deben tomarse con precaución y de forma complementaria a otros, con el fin de evitar errores en el diagnóstico (Hanley et al., 2009; Mori y Christodoulou, 2012).

\section{CONCLUSIONES}

Como contribución a la evaluación de la sostenibilidad de sistemas socioecológicos como las islas turísticas, se presenta un modelo dinámico integral y algunas de sus aplicaciones. El modelo de sostenibilidad de Fuerteventura (MSF), calibrado para el período 1996-2011, ha facilitado un análisis dinámico de este sistema socioecológico, así como la identificación y cuantificación de interacciones que se producen entre diferentes indicadores de sostenibilidad, que podrían haber pasado inadvertidas si sólo se utilizan catálogos estáticos de indicadores.

Además, se ha aplicado el MSF para el análisis del comportamiento de 6 indicadores bajo un conjunto de escenarios y medidas, durante el período 2012-2025. Al final de este período, tres de los seis indicadores $(E P p c, C O 2 p c$ e $I A E)$ excederían sus umbrales en todas las simulaciones y uno (TURES), en casi todas. En los escenarios de cambio climático A2 y B2, dos de los seis indicadores $(E P p c, C O 2 p c)$ empeorarían, en relación con ESC. En cuanto a las medidas de gestión, M.I -toda la demanda energética de desalinización proporcionada por fuentes renovables- mejoraría los resultados de los tres indicadores energéticos, aunque aún lejos de sus umbrales de sostenibilidad (EPpc, CO2pc e IAE). Con la implantación de la medida II -limitación de la construcción de nuevos alojamientos turísticos- dos indicadores mejorarían en comparación con ESC (RATPR y PSA; IAE no sufre cambios), mientras que tres (TURES, EPpc y CO2pc) empeorarían. En relación al comportamiento de estos tres últimos, cabe señalar que en este trabajo ha quedado reflejado que algunos indicadores relativos no siempre brindan información sólida sobre la sostenibilidad cuando se consideran de manera aislada, o con un valor discreto, estático. Por ello hay que evaluar su evolución y las interacciones con otras variables (Hanley et al., 2009) para evitar errores en el diagnóstico.

Estos resultados preliminares también sugieren que el sistema socioecológico de Fuerteventura podría ser más reactivo a ciertas medidas de gestión (como la Medida II) 
que a los factores externos, tanto climáticos como económicos. Por ejemplo, mientras que bajo el escenario de recesión, indicadores como PSA mejorarían alrededor de 3\%; bajo la medida de gestión M. II, esta mejora alcanzaría casi el 24\%. Esto refleja el impacto de las acciones (y las inacciones) de las políticas en la gestión de este SSE y subraya la responsabilidad de los que toman decisiones a la hora de abordar medidas para contribuir a un desarrollo más equilibrado y sostenible del sistema socioecológico de Fuerteventura.

Por otro lado, también se ha identificado que en el Plan de Acción faltan medidas más ambiciosas para alcanzar los objetivos de sostenibilidad con respecto a los problemas del binomio agua-energía en esta isla. Además, en este trabajo se muestra la utilidad de estas herramientas (la simulación dinámica combinada con indicadores de sostenibilidad y sus umbrales, así como la modelización de escenarios y medidas) para el diagnóstico de la sostenibilidad en destinos turísticos como Fuerteventura y para la toma de decisiones al respecto.

\section{BIBLIOGRAFÍA}

ALCAMO, J., FLÖRKE, M. y MÄRKER, M. (2007): «Future long-term changes in global water resources driven by socio-economic and climatic changes», Hydrological Sciences Journal, vol. 52 (2), pp. 247-275. DOI: 10.1623/hysj.52.2.247

ANDARIZAN, B., BANNAYANB, M., STEDUTOC, P., MAZRAEHAM H., BARATID, M.E., BARATIE, M.A. y RAHNAMA, A. (2011): «Validation and testing of AquaCrop model under full and deficit irrigated wheat production in Iran», Agricultural Water Management, vol. 100 (1), pp. 1-8.

ANDERIES, J.M., JANSSEN M.A. y OSTROM, E. (2004): «A framework to analyze the robustness of social-ecological systems from an institutional perspective», Ecology and Society, vol. 9 (1), pp. 18.

AP (PLAN DE ACCIÓN DE LA RESERVA DE LA BIOSFERA DE FUERTEVENTURA). (2013). Disponible en http://gestion.cabildofuer.es/fuerteventurabiosfera/

BANOS-GONZÁLEZ, I., MARTÍNEZ-FERNÁNDEZ, J. y ESTEVE, M.A. (2015): «Dynamic integration of sustainability indicators in insular socio-ecological systems», Ecological Modelling, no 306 (Special Issue: Ecological Modelling for Ecosystem Sustainability), pp.130-144.

BANOS-GONZÁLEZ, I. (2016): Integración dinámica de indicadores de sostenibilidad en el modelo socioecológico de la Reserva de la Biosfera de Fuerteventura. Tesis doctoral: http://fobos.inf.um.es/ecomed/?p=660

BARLAS, Y. (1996): «Formal aspects of model validity and validation in system dynamics», System dynamics review, vol. 12 (3), pp. 183-210.

BECKEN, S., SIMMONS, D.G. y FRAMPTON, C. (2003): «Energy use associated with different travel choices», Tourism Management, vol. 24 (3), pp. 267-277.

BLANCO, J.A. (2013): «Modelos ecológicos: descripción, explicación y predicción». Ecosistemas, vol. 22 (3), pp.1-5. DOI: 10.7818/ECOS.2013.22-3.01.

BPIA, (2012): «Banco Público de Indicadores Ambientales del MAGRAMA». Disponible en http://www.magrama.gob.es/es/calidad-y-evaluacion-ambiental/temas/informacion- 
ambiental-indicadores-ambientales/banco-publico-de-indicadores-ambientales-bpia-/ default.aspx

CABRERA, M.C. y CUSTODIO, E. (2012): «The Canary Islands», en Water, agriculture and the environment in Spain: Can we square the circle? L. De Stefano \& M.R. Llamas (Eds.), CRC Press. Taylor and Francis, pp. 281-291.

CÁCERES, Y. (2010): Sustainability indicators system for Fuerteventura Biosphere Reserve. Fuerteventura Biosphere Reserve.

CANALIS, X. (2013): «La Primavera Árabe beneficia realmente a España?», Destinos turísticos y Geopolítica, pp. 38-9. Disponible en https://www.hosteltur.com/137410_ primavera-arabe-beneficia-realmente-espana.html

CEDEX [Centro de Estudios y Experimentación de Obras Públicas] (2011): «Evaluación del impacto del cambio climático en los recursos hídricos en régimen natural. Encomienda de Gestión de la Dirección General del Agua al CEDEX para el estudio del cambio climático en los recursos hídricos y las masas de agua», Ministerio de Medio Ambiente y Medio Rural y Marino. Disponible en http://www.magrama.gob.es/es/ cambio-climatico/publicaciones/publicaciones/Memoria_encomienda_CEDEX_tcm7165767.pdf

CHESTER, L. (2010): «Conceptualising energy security and making explicit its polysemic nature», Energy policy, vol. 38 (2), pp. 887-895. DOI: 10.1016/j.enpol.2009.10.039

CIAFV (Fuerteventura Island Water Council). (2009). Disponible en http://www.aguasfuerteventura.com/

CRUZ, A. (2009): «Tourist attraction factors: similarities and differences in major destinations in the Caribbean Islands», TURyDES, vol. 2 (6), pp. 1-18.

DENIS, G.S. y PARKER, P. (2009): «Community energy planning in Canada: The role of renewable energy», Renewable and Sustainable Energy Reviews, vol. 13 (8), pp. 2.088-2.095.

DEYÀ TORTELLA, B. y TIRADO, D. (2011): «Hotel water consumption at a seasonal mass tourist destination. the case of the island of Mallorca», Journal of Environmental Management, vol. 92 (10), pp. 2568-2579. doi:10.1016/j.jenvman.2011.05.024

DODDS, R. (2007): «Malta's tourism policy: standing still or advancing towards sustainability», Island Studies Journal, vol. 2 (1), pp. 47-66.

DUFFI, A. (2001): A trip too far. Ecotourism, politics and exploitation. Earthscan, London

EC (2008): European Commission. Communication from the Commission-energy efficiency: delivering the 20\% target. COM 2008, 772 final.

EC (2015): «European Commission, Climate Action», EU Action 2030. Disponible en http://ec.europa.eu/clima/policies/2030/index_en.htm

FILATOVA, T. y POLHILL, G. (2012): «Shocks in Coupled Socio-ecological Systems: What Are They and How Can We Model Them? International Environmental Modelling and Software Society (iEMSs)», International Congress on Environmental Modelling and Software Managing Resources of a Limited Planet, Sixth Biennial Meeting, Leipzig, Germany.

FOLKE, C., HAHN, T., OLSSON, P. y NORBERG, J. (2005): «Adaptive governance of social-ecological systems», Annual Review of Environmental Resources, vol. 30, 441-473. 
FONG, W.K., MATSUMOTO, H. y LUN, Y.F. (2009): «Application of System Dynamics model as decision making tool in urban planning process toward stabilizing carbon dioxide emissions from cities», Building and Environment, vol. 44 (7), pp. 1528-1537. FORRESTER, J.W. (1961): Industrial dynamics. Waltham MA: Pegasus Communications. GALLOPIN, G.C. (1997): «Indicators and their use: information for decision-making», en Sustainability indicators: Report on the project on indicators of sustainable development. B. Moldan, S. Billharz \& R. Matravers (Eds.), SCOPE 58. Chichester: John Wiley \& Sons.

GARÍN-MUÑOZ, T. (2006): «Inbound international tourism to Canary Islands: a dynamic panel data model», Tourism Management, vol. 27 (2), pp. 281-291.

GOH, C., LAW, R. (2002): «Modeling and forecasting tourism demand for arrivals with stochastic nonstationary seasonality and intervention», Tourism Management, vol. 23(5), pp. 499-510.

GOBIERNO DE CANARIAS (2003): «Ley 19/2003 de 14 de abril, por la que se aprueban las Directrices de Ordenación General y las Directrices de Ordenación del Turismo de Canarias». Boletín Oficial de Canarias vol. 73, 15/04/2003, pp. 5662-5779.

GOBIERNO DE CANARIAS (2008): «Metodología para la aplicación práctica de la apreciación y evaluación de los factores determinantes de la capacidad de carga. Especialmente en zonas turísticas», Gobierno de las Islas Canarias. Disponible en http://www.fecam.es/documentos/areas/turismo_transportes/CCTGuia.pdf

GOBIERNO DE CANARIAS (2010): «Pasajeros por vía aérea procedentes del extranjero», Viceconsejería de Turismo. Estadísticas y Estudios. Disponible en http://www. gobiernodecanarias.org/presidencia/turismo/estadisticas_y_estudios/Pasajeros_procedentes_del_extranjero_segxn_Pais_de_origen_/index-bis.html

GRAHAM, A.K., MOORE, J. y CHOI, C.Y. (2002): «How robust are conclusions from a complex calibrated model, really? A project management model benchmark using fit-constrained Monte Carlo analysis», Proceedings of the XX System Dynamics Conference. System Dynamics Society. Palermo. 28 July-1 August 2002.

GRAYMORE, M.L., SIPE, N.G. y RICKSON, R.E. (2010): «Sustaining human carrying capacity: a tool for regional sustainability assessment», Ecological Economics, vol. 69 (3), pp. 459-468.

GUNDERSON, L.H. y HOLLING, C.S. (2002): Panarchy: understanding transformations in systems of humans and nature. Island, Washington.

HALLIDAY, A. Y GLASER, M. (2011): «A Management Perspective on Social Ecological Systems: A generic system model and its application to a case study from Peru», Human Ecology Review, vol. 18 (1), pp. 1-18.

HANLEY, N., MCGREGOR, P.G., SWALES, J.K. y TURNER, K. (2009): «Do increases in energy efficiency improve environmental quality and sustainability?», Ecological Economics, vol. 68 (3), pp. 692-709.

HERRERA, C. y CUSTODIO, E. (2000): «Saline water in Central Fuerteventura Island, Canary Islands, Spain», en XV Salt Water Intrusion, Meeting, Miedzyzdroje-Wolin Island, vol. 80 (1), pp. 81-86.

HERNÁNDEZ- LUIS, J.A., GONZÁLEZ MORALES, A., HERNÁNDEZ TORRES, S. y RAMÓN OJEDA, A.A. (2017): «El impacto del turismo de masas en las Islas Canarias 
en el contexto de las reservas mundiales de la biosfera», Cuadernos de Turismo, $\mathrm{n}^{\circ}$ 40, pp. 363-387.

HODBOD, J. y ADGER, W.N. (2014): «Integrating social-ecological dynamics and resilience into energy systems research», Energy Research \& Social Science, vol. 1, pp. 226-231.

HJORTH, P. y BAGHERI, A. (2006): «Navigating towards sustainable development: A system dynamics approach», Futures, vol. 38 (1), pp. 74-92. DOI: 10.1016/j. futures.2005.04.005

IGLESIAS, G. y CARBALLO, R. (2011): «Wave resource in El Hierro: an island towards energy self-sufficiency», Renewable Energy, 36 (2), pp. 689-698.

IPCC (2014): «Fifth Assessment Report (AR5)», Disponible en https://www.ipcc.ch/ report/ar5/index.shtml

ISTAC (2012): «Instituto Canario de Estadística (1998-2012)», Disponible en http://www. gobiernodecanarias.org/istac/temas_estadisticos/empleo/empleo/actividadeconomica/ E30308A.html

JANSSEN, M.A. (2006): «Historical institutional analysis of social-ecological systems», Journal of Institutional Economics, vol. 2 (2), pp. 127-131.

JOHANSSON, T.B. y GOLDEMBERG, J. (2004): «Energy and the Challenge of Sustainability Overview: 2004 Update, United Nations Development Programme», United Nations Department of Economic and Social Affairs, and World Energy Council. Disponible en http://www.undp.org/seed/eap/activities/wea/

JØRGENSEN, S.E. (2013): «The development of a carbon cycling model for the Danish island of Sams $\varnothing$ based on renewable energy», 19th ISEM Conference on Ecological Modelling for Ecosystem Sustainability in the context of Global Change. Toulouse, 28-31.

KAJIKAWA Y, INOUE T. y GOH T.N. (2011): «Analysis of building environment assessment frameworks and their implications for sustainability indicators», Sustainability Science, vol. 6 (2), pp. 233-46.

KELLY, R.A., JAKEMAN, A.J., BARRETEAU, O., BORSUK, M.E., ELSAWAH, S., HAMILTON, S.H. y VOINOV, A.A. (2013): «Selecting among five common modelling approaches for integrated environmental assessment and management», Environmental Modelling \& Software, vol. 47, pp. 159-181.

KO TG. (2005): «Development of a tourism sustainability assessment procedure: a conceptual approach», Tourism Management, vol. 26 (3), pp. 431-445. DOI: 10.1016/j. tourman.2003.12.003

KRUYT, B., VAN VUUREN, D.V., DE VRIES, H.J.M. y GROENENBERG, H. (2009): «Indicators for energy security», Energy Policy, vol. 37 (6), pp. 2.166-2.181.

KUO, N.W. y CHEN, P.H. (2009): «Quantifying energy use, carbon dioxide emission, and other environmental loads from island tourism based on a life cycle assessment approach», Journal of Cleaner Production, vol. 17 (15), pp. 1.324-1.330.

LANCKER, E. y NIJKAMP, P. (2000): «A policy scenario analysis of sustainable agricultural development options: a case study for Nepal», Impact Assessment and Project Appraisal, vol. 18 (2), pp. 111-124. DOI: 10.3152/147154600781767493 
LI, F.J., DONG, S.C., LI, F. (2012): «A system dynamics model for analyzing the ecoagriculture system with policy recommendations», Ecological Modelling, vol. 227 (1), pp. 34-45.

MARTÍNEZ-MOYANO, I.J. y RICHARDSON, G.P. (2013): «Best practices in system dynamics modeling», System Dynamics Review, vol. 29 (2), pp. 102-123.

MATOS, C., TEIXEIRA, C.A., BENTO, R., VARAJÃO, J. y BENTES, I. (2014): «An exploratory study on the influence of socio-demographic characteristics on water end uses inside buildings», Science of the Total Environment, vol. 466, pp. 467-474. DOI: 10.1016/j.scitotenv.2013.07.036

MOLDAN, B., JANOUŠKOVÁ, S. y HÁK, T. (2012): «How to understand and measure environmental sustainability: Indicators and targets», Ecological Indicators, vol. 17 (1), pp. 4-13.

MORI, K. y CHRISTODOULOU, A. (2012): «Review of sustainability indices and indicators: towards a new City Sustainability Index (CSI)», Environmental Impact Assessment Review, vol. 32 (1), pp. 94-106.

NIELSEN, S.N. y JØRGENSEN, S.E. (2015): «Sustainability analysis of a society based on exergy studies-a case study of the island of Samsø (Denmark)», Journal of Cleaner Production, vol. 96, pp. 12-29. DOI: 10.1016/j.jclepro.2014.08.035

OIKONOMOU, E.K., KILIAS, V., GOUMAS, A., RIGOPOULOS, A., KARAKATSANI, E., DAMASIOTIS, M. y MARINI, N. (2009): «Renewable energy sources (RES) projects and their barriers on a regional scale: The case study of wind parks in the Dodecanese islands, Greece», Energy Policy, vol. 37 (11), pp. 4874-4883. DOI: 10.1016/j. enpol.2009.06.050

OLIVA, R. (2003): «Model calibration as a testing strategy for system dynamics models», European Journal of Operational Research, vol. 151(3), pp. 552-568.

OREJA-RODRÍGUEZ, J.R, PARRA-LÓPEZ, E. y YANES-ESTÉVEZ, V. (2008): «The sustainability of island destinations: Tourism area life cycle and teleological perspectives. The case of Tenerife», Tourism Management, vol. 29 (1), pp. 53-65. DOI:10.1016/j. tourman.2007.04.007

OSTROM, E. (2009): «Institutional Rational Choice: An Assessment of the Institutional Analysis and Development Framework», en: Theories of the Policy Process, 2nd ed., ed. PaulSabatier. Boulder, CO: Westview Press, 21-64.

PALACIOS, M.P., MENDOZA-GRIMÓN, V., FERNÁNDEZ-VERA, J.R., RODRÍGUEZ-RODRÍGUEZ, F., TEJEDOR-JUNCO, M.T. y HERNÁNDEZ-MORENO, J.M. (2009): «Subsurface drip irrigation and reclaimed water quality effects on phosphorus and salinity distribution and forage production», Agricultural water management, vol. 96(11), pp. 1659-1666. DOI: 10.1016/j.agwat.2009.06.021

PETROSILlO, I., COSTANZA, R., ARETANO, R., ZACCARELLI, N. y ZURLINI, G. (2013): «The use of subjective indicators to assess how natural and social capital support residents' quality of life in a small volcanic island», Ecological Indicators, vol. 24, pp. 609-620.

PNUMA. 2002: «Perspectivas del Medio Ambiente Mundial 2002 GEO-3», Disponible en https://web.unep.org/geo/sites/unep.org.geo/files/documents/prelims_es.pdf 
POVEDA, C.A., y LIPSETT, M.G. (2014): «An integrated approach for sustainability assessment: the Wa-Pa-Su project sustainability rating system», International Journal of Sustainable Development \& World Ecology, vol. 21(1), pp. 85-98. DOI: 10.1080/13504509.2013.876677

QIN, H.P., SU, Q. y KHU, S.T. (2011): «An integrated model for water management in a rapidly urbanizing catchment», Environmental Modelling \& Software, vol. 26(12), pp. 1502-1514. DOI: 10.1016/j.envsoft.2011.07.003

REED, M.S., FRASER, E.D. y DOUGILL, A.J. (2006): «An adaptive learning process for developing and applying sustainability indicators with local communities», Ecological economics, vol. 59(4), pp. 406-418. DOI: 10.1016/j.ecolecon.2005.11.008

SANTANA-JIMÉNEZ, Y. y HERNÁNDEZ, J.M. (2011): «Estimating the effect of overcrowding on tourist attraction: The case of Canary Islands», Tourism Management, vol. 32(2), pp. 415-25.

SANTANA-JIMENEZ, Y., HERNANDEZ, J.M. y SUAREZ-VEGA, R. (2017): «Land use conversion from tourist to residential», Current Issues in Tourism, pp. 1-19.

SCHALDACH, R., KOCH, J., DER BEEK, T.A., KYNAST, E. y FLÖRKE, M. (2012): «Current and future irrigation water requirements in pan-Europe: An integrated analysis of socio-economic and climate scenarios», Global and Planetary Change, vol. 94, pp. 33-45. DOI: 10.1016/j.gloplacha.2012.06.004

SCHUSTER, C., IGLESIAS-LEBRIJA, J.J. y CARRASCAL, L.M. (2012): «Recent population trends of the houbara bustard in the Canary Islands. Methods and conservation status», Animal Biodiversity and Conservation, vol. 35 (1), pp.125-39.

SEPASKHAH, A.R., AMINI-NEJAD, M. y KAMGAR-HAGHIGHI, A.A. (2013): «Developing a dynamic yield and growth model for saffron under different irrigation regimes», International Journal of Plant Production, vol. 7 (3), pp. 473-504.

SINGH, R.K., MURTY, H.R., GUPTA, S.K. y DIKSHIT, A.K. (2012): «An overview of sustainability assessment methodologies», Ecological Indicators, vol. 15(1), pp. 281299. DOI: 10.1016/j.ecolind.2011.01.007

SPILANIS I, KIZOS T, KOULOURI M, KONDYLI J., VAKOUFARIS H. y GATSIS I. (2009): «Monitoring sustainability in insular areas», Ecological Indicators, vol. 9(1), pp. 179-87. DOI: 10.1016/j.ecolind.2008.03.003

TORRES CABRERA, J.M. (1995): The soil as a natural resource: degradation process and their impact on desertification of the island of Fuerteventura. Unpublished dissertation. La Laguna University, Spain.

UNESCO (2009): Disponible en http://www.unesco.org/new/en/natural-sciences/environment/ ecological-sciences/biosphere-reserves/europe-north-america/spain/fuerteventura/

TSOUKALA, A., SPILANIS, I., TSIRTSIS, G., BANOS-GONZÁLEZ, I, MARTÍNEZ, J. y ESTEVE, M.A (2018): «An integrated framework for decision support in islands' management: a case-study for a 'typical' Mediterranean island», Island Studies Journal, vol. 23, $\mathrm{n}^{\mathrm{o}} 2$ (en prensa).

Ventana System. (2011): «Vensim $® »$, Disponible en http://www.vensim.com 
VOINOV, A. y SHUGART, H.H. (2013): «〈Integronsters», integral and integrated modeling», Environmental Modelling \& Software, vol. 39, pp. 149-158. DOI: 10.1016/j. envsoft.2012.05.014

VON GEIBLER, J., KRISTOF, K. y BIENGE, K. (2010): «Sustainability assessment of entire forest value chains: Integrating stakeholder perspectives and indicators in decision support tools», Ecological Modelling, vol. 221 (18), pp. 2206-2214.DOI: 10.1016/j.ecolmodel.2010.03.022

WCED [World Commission on Environment and Development] (1987): Our Common Future. Oxford University Press, Oxford. 